\title{
Hyperthermia accelerates neuronal loss differently between the hippocampal CA1 and CA2/3 through different HIF-1 $\alpha$ expression after transient ischemia in gerbils
}

\author{
TAE-KYEONG LEE ${ }^{1 *}$, DAE WON KIM ${ }^{2 *}$, HYEJIN SIM ${ }^{3}$, JAE-CHUL LEE $^{3}$, \\ HYUNG IL KIM ${ }^{4,5}$, MYOUNG CHEOL SHIN ${ }^{4}$, JUN HWI CHO ${ }^{4}$, JOON HA PARK ${ }^{6}$, \\ CHOONG-HYUN LEE ${ }^{7}$, MOO-HO WON ${ }^{3}$ and JI HYEON AHN ${ }^{3,8}$
}

${ }^{1}$ Department of Food Science and Nutrition, Hallym University, Chuncheon, Gangwon 24252;

${ }^{2}$ Department of Biochemistry and Molecular Biology and Research Institute of Oral Sciences, College of Dentistry, Gangnung-Wonju National University, Gangneung, Gangwon 25457; ${ }^{3}$ Department of Neurobiology, School of Medicine, Kangwon National University, Chuncheon, Gangwon 24341; ${ }^{4}$ Department of Emergency Medicine,

Kangwon National University Hospital, School of Medicine, Kangwon National University, Chuncheon, Gangwon 24289;

${ }^{5}$ Department of Emergency Medicine, Dankook University Hospital, College of Medicine, Dankook University, Cheonan, Chungnam 31116; ${ }^{6}$ Department of Anatomy, College of Korean Medicine, Dongguk University, Gyeongju, Gyeongbuk 38066;

${ }^{7}$ Department of Pharmacy, College of Pharmacy, Dankook University, Cheonan, Chungnam 31116; ${ }^{8}$ Department of Physical Therapy, College of Health Science, Youngsan University, Yangsan, Gyeongnam 50510, Republic of Korea

Received January 4, 2022; Accepted February 17, 2022

DOI: $10.3892 /$ ijmm.2022.5111

\begin{abstract}
The hippocampus has a different vulnerability to ischemia according to the subfields CA1 to CA3 (initials of cornu ammonis). It has been reported that body temperature changes during ischemia affect the degree of neuronal death following transient ischemia. Hypoxia-inducible factor $1 \alpha$ (HIF-1 $\alpha$ ) plays a key role in regulating cellular adaptation to low oxygen conditions. In the present study, we investigated the pattern of neuronal death (loss) in CA1 and CA2/3 following 5 min transient forebrain ischemia (TFI) under hyperthermia $\left(39.5 \pm 0.2^{\circ} \mathrm{C}\right)$ and the relationship between neuronal death and changes in HIF-1 $\alpha$ expression using western blot analysis and immunohistochemistry in gerbils. Normothermia or
\end{abstract}

Correspondence to: Professor Moo-Ho Won, Department of Neurobiology, School of Medicine, Kangwon National University, 1 Kangwondaehak-gil, Chuncheon, Gangwon 24341, Republic of Korea

E-mail: mhwon@kangwon.ac.kr

Professor Ji Hyeon Ahn, Department of Physical Therapy, College of Health Science, Youngsan University, 288, Junam-ro, Yangsan, Gyeongnam 50510, Republic of Korea

E-mail: jh-ahn@ysu.ac.kr

*Contributed equally

Key words: body temperature, hippocampal subfields, low oxygen, pyramidal neurons, resistance to ischemia, transient forebrain ischemia hyperthermia was induced for $30 \mathrm{~min}$ before and during the TFI, and neuronal death and HIF-1 $\alpha$ expression were observed at $0,3,6$ and $12 \mathrm{~h}, 1,2$ and 5 days after TFI. Under normothermia, TFI-induced neuronal death of CA1 pyramidal neurons occurred on day 5 after TFI, but CA2/3 pyramidal neurons did not die. In contrast, under hyperthermia, the death of CA1 and CA2/3 pyramidal neurons was observed on day 2 after TFI. Under normothermia, HIF-1 $\alpha$ expression was significantly elevated in both $\mathrm{CA} 1$ and $\mathrm{CA} 2 / 3$ pyramidal neurons at $12 \mathrm{~h}$ and 1 day after TFI, and the increased HIF-1 $\alpha$ immunoreactivity in CA1 was dramatically reduced from 2 days after TFI, but not in CA2/3 pyramidal neurons. Under hyperthermia, the basal expression of HIF-1 $\alpha$ in the sham group was significantly higher in both CA1 and CA2/3 pyramidal neurons at $0 \mathrm{~h}$ after TFI than in the normothermia group. HIF-1 expression was continuously higher, peaked at $12 \mathrm{~h}$ after TFI, and then significantly decreased from 1 day after TFI. Overall, the present results indicate that resistance to ischemia in CA2/3 pyramidal neurons is closely associated with the persistence of increased expression of HIF-1 $\alpha$ after ischemic insults and that hyperthermia-induced exacerbation of death of pyramidal neurons is closely related to decreased HIF-1 $\alpha$ expression after ischemic insults.

\section{Introduction}

It is well known that cerebral neurons have different susceptibilities to deleterious conditions, such as ischemic injury and neurodegeneration, depending on brain area (1). The hippocampus is one of the brain areas vulnerable to ischemic insults, and hippocampal neurons respond differently to ischemic damage according to its subfields (CA1-3) and impact of 
ischemia. A short period (5 min) of transient forebrain ischemia (TFI) in gerbils induces death of pyramidal neurons located in hippocampal CA1 at 4 or 5 days after TFI, but pyramidal neurons of CA2/3 are resistant $(2,3)$. Hippocampal neuronal vulnerability to cerebral ischemia can be altered by body temperature or ischemic duration $(4,5)$. Higher temperatures cause more severe ischemic damage. It was reported that in gerbils, hyperthermia before and during a brief episode (5 min) of TFI resulted in the accelerated death of hippocampal CA1 pyramidal neurons (6). Clinically, increased body temperature during the first day after stroke is related to poor outcomes and leads to devastating effects on mortality (7).

Hypoxia-inducible factor $1 \alpha$ (HIF- $1 \alpha$ ), a well-known isoform of hypoxia-inducible factors, exerts hypoxia-inducible transcriptional activity triggered by low oxygen conditions, whereas HIF-1 $\beta$ is an oxygen-insensitive subunit (8). HIF-1 $\alpha$ expression is localized in the cytosol of pyramidal neurons located in all hippocampal subfields (CA1-3) of rats and gerbils (9-11). HIF-1 $\alpha$ plays a key role in regulating cellular adaptation to low oxygen conditions by modulating gene expression, targeting cell survival, angiogenesis, and glucose metabolism, which may contribute to alleviating ischemic neuronal damage $(12,13)$. Increased HIF-1 $\alpha$ expression significantly attenuates post-ischemic damage of hippocampal CA1 pyramidal neurons by increasing the expression of nuclear factor- $\kappa \mathrm{B}(\mathrm{NF}-\kappa \mathrm{B})$ and vascular endothelial growth factor (VEGF) following ischemic preconditioning (9). In addition, systemic administration of a HIF-1 $\alpha$ activator (ML228) was found to alleviate neuronal apoptosis in hippocampal CA1 by attenuating the amplified expression of pro-inflammatory cytokines and their receptors following cardiac arrest-induced cerebral ischemia (14).

Although previous studies have reported the effects of HIF-1 $\alpha$-mediated neuroprotection and its related signaling pathways, few studies have been conducted on changes in HIF-1 $\alpha$ expression in hippocampal subfields induced by TFI under hyperthermic conditions that cause more severe neuronal damage. Therefore, the present study aimed to investigate the pattern of neuronal death and expression of HIF-1 $\alpha$ in hippocampal subfields (CA1-3) after 5 min TFI under hyperthermic conditions in gerbils.

\section{Materials and methods}

Experimental animals and groups. In the present study, a total of 240 male gerbils (age, 6 months; body weight, $70 \pm 5 \mathrm{~g}$ ) were used at the start of the experiment. The gerbils were bred and kept in the Experimental Animal Center at Kangwon National University located in Chuncheon (Korea). The animals were housed in a conventional room (temperature, $22 \pm 2^{\circ} \mathrm{C}$; humidity, $57 \pm 5 \%$; light/dark cycle, 12:12) with freely accessible water and food. All experimental procedures were approved (approval no. KW-200113-1) by the Institutional Animal Care and Use Committee on January 13, 2020. The Animal care and handling conformed to the NIH Guide for the Care and Use of Laboratory Animals (The National Academies Press, 8th edition, 2011) (15). All efforts were taken at each stage of the experiments to minimize the numbers of animals used and to limit any discomfort to which the animals might be exposed.
Experimental gerbils (total $n=240$ ) were divided into four groups: i) sham-operated gerbils under normothermia (NT/sham group; $n=36$ ), ii) TFI-operated gerbils under normothermia (NT/ischemia group; $n=84)$, iii) sham-operated gerbils under hyperthermia (HT/sham group; n=36), iv) TFI-operated gerbils under hyperthermia (HT/ischemia group; $n=84$ ).

In each group, 12 gerbils ( $n=5$ for western blotting; $n=7$ for histology) were sacrificed at $0 \mathrm{~h}$ (immediately after $5 \mathrm{~min}$ of TFI operation), 3, 6 and 12 h, 1, 2 and 5 days after TFI (Fig. 1).

Induction of TFI under normothermia or hyperthermia. TFI induction was performed according to previously described methods $(16,17)$ with minor modification. The gerbils were anesthetized using inhalation anesthesia machine (Harvard Apparatus) with isoflurane (2.5\%; Baxtor) in oxygen $(33 \%)$ and nitrous oxide $(67 \%)$. Under anesthesia, the bilateral common carotid arteries, which are located in the ventral neck, were ligated with aneurysm clips for $5 \mathrm{~min}$. Before and during the TFI surgery, body temperature was controlled using a heating pad, which was connected to a rectal thermistor (Harvard Apparatus) (Fig. 1). Rectal temperature was controlled at $37.0 \pm 0.5^{\circ} \mathrm{C}$ for normothermia and elevated up to $39.5 \pm 0.2^{\circ} \mathrm{C}$ for hyperthermia for $30 \mathrm{~min}$. After the TFI surgery, the gerbils were kept in thermal incubators (temperature, $22 \pm 2^{\circ} \mathrm{C}$; humidity, $57 \pm 5 \%$ ) to adjust body temperature on a normothermic level. The gerbils in the sham groups with normothermia or hyperthermia received the same TFI surgery without ligation of the arteries.

Western blot analysis. To analyze HIF-1 $\alpha$ protein level in CA1 and CA2/3, the gerbils of each group were sacrificed under profound anesthesia by intraperitoneal (i.p.) injection of pentobarbital sodium (200 mg/kg) (18) (JW Pharmaceutical Co., Ltd.) at the designated time $(0,3,6$ and $12 \mathrm{~h}, 1,2$ and 5 days after TFI). The death of animals was confirmed according to vital signs including heart beats, pupillary response, and respiratory pattern (lack of cardiac activity for 5 min by cardiac palpation, unresponsiveness to light with dilated pupils using light into the eyes of the animal, and lack of spontaneously breathing pattern with shallow and irregular breathing pattern). CA1 and CA2/3 tissues were respectively collected from the hippocampi using brain matrix. Proteins of CA1 and CA2/3 were extracted according to a previously published method (9) using rabbit anti-HIF-1 $\alpha$ (cat. no. ab228649, diluted 1:5,000, Abcam) and anti- $\beta$-actin (cat. no. ab8227, diluted 1:20,000, Abcam) as primary antibodies.

For quantification of the bands, densitometry analysis was performed using Scion Image software, Ver 4.0 (Scion Corp.). The ratio, as relative density, of HIF- $1 \alpha$ protein was calibrated with the corresponding expression rate of $\beta$-actin and normalized to that in NT/sham group at $0 \mathrm{~h}$.

Tissue processing for histology. For histology, the gerbils of each group were profoundly anesthetized with $200 \mathrm{mg} / \mathrm{kg}$ (i.p.) of pentobarbital sodium (18) (JW Pharmaceutical Co., Ltd.) at the designated time $(0,3,6$ and $12 \mathrm{~h}, 1,2$ and 5 days after TFI) and sacrificed after confirmation of death according to the method described above. As previously described $(16,17)$, in short, the gerbils were perfused transcardially with 

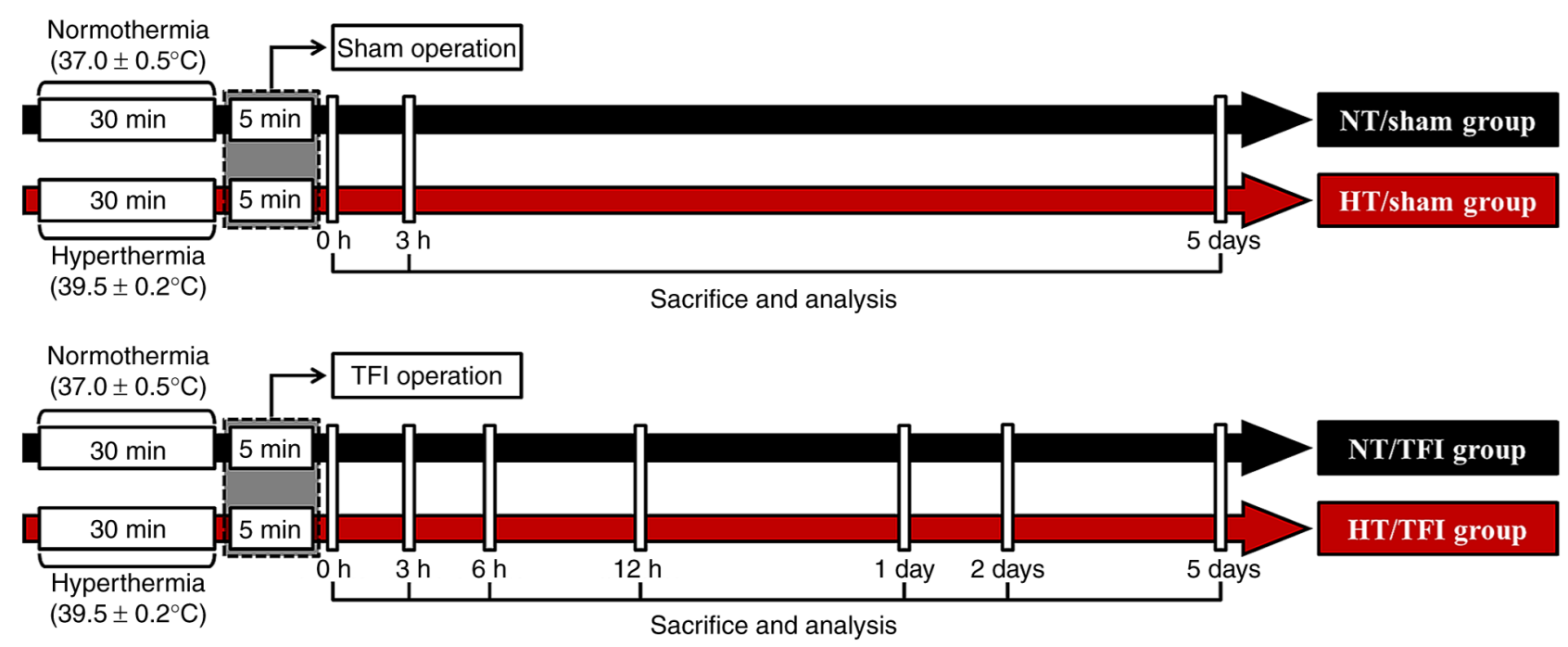

Figure 1. Timeline of the experiment. Body temperature was controlled before TFI for 30 min and during TFI. The gerbils of the sham groups were sacrificed for western blot and histological analyses at 0 and $3 \mathrm{~h}$ and 5 days, and those of the TFI groups were sacrificed at $0,3,6$ and $12 \mathrm{~h}, 1,2$ and 5 days after TFI. TFI, transient forebrain ischemia.

4\% paraformaldehyde and their brains were extracted from the skulls. The brains were post-fixed in the same fixative for $5 \mathrm{~h}$. The fixed brain tissues were infiltrated with $30 \%$ sucrose as cryoprotective agent when the brain tissues were cut. To prepare brain sections containing the hippocampus, using a cryostat, the frozen tissues were frontally cut into a $30-\mu \mathrm{m}$ thickness. Thereafter, the sections were stored in 6-well plates containing PBS for histological staining.

Histochemistry using cresyl violet $(C V)$. To examine the morphology and distribution of hippocampal cells in all groups, CV histochemical staining was conducted as previously described (9). In brief, the sections were stained with $1.0 \% \mathrm{CV}$ acetate (Sigma-Aldrich; Merck KGaA) containing $0.28 \%$ glacial acetic acid. The stained sections were dehydrated using ethanol series and mounted with Canada balsam.

For analysis of hippocampal change following TFI, CV-stained structures were observed using AxioM1 light microscope at $\mathrm{x} 4$ and $\mathrm{x} 20$ magnifications (Carl Zeiss). In this examination, six sections per gerbil were selected at $120-\mu \mathrm{m}$ intervals.

Histofluorescence using Fluoro-Jade B (FJB). To examine TFI-induced neuronal death (loss) in CA1 and CA2/3, histofluorescence using FJB, a representative marker of neuronal degeneration/death, was conducted according to previously published studies $(16,17)$. In brief, the brain tissues were soaked in $0.06 \%$ potassium permanganate and incubated in $0.0004 \%$ FJB (Histochem). Thereafter, the stained sections were prepared for observation.

For analysis of FJB-positive cells, images of FJB-positive cells were captured using a fluorescence microscope (Carl Zeiss) with blue excitation fluorescence filter (450-490 nm). The captured images (FJB-positive cells) were analyzed using image analyzing software (NIH Image J 1.59). Cell count was performed in $400 \mu \mathrm{m}^{2}$ at middle of the stratum pyramidale of CA 1 and CA2/3.
Immunohistochemistry. To examine changes in neurons and HIF-1 $\alpha$ expression, immunohistochemistry was performed according to previously published methods $(9,19,20)$. In short, the brain sections were immersed in $0.3 \%$ hydrogen peroxide $\left(\mathrm{H}_{2} \mathrm{O}_{2}\right)$ and soaked in 5\% normal goat serum. Thereafter, the brain sections were incubated with each primary antibody, rabbit anti-neuronal nuclei (cat. no. MAB377, NeuN; diluted 1:1,100, Chemicon International) and rabbit anti-HIF-1 $\alpha$ (cat. no. ab8366, diluted 1:500, Abcam) overnight at $4^{\circ} \mathrm{C}$. Subsequently, they were incubated in secondary antibodies, biotinylated goat anti-rabbit IgG (cat. no. BA-1000-1.5, diluted 1:200, Vector Laboratories, Inc.) and streptavidin peroxidase complex (diluted 1:200, Vector Laboratories, Inc.). Finally, the sections were visualized using $0.05 \%$ 3,3'-diaminobenzidine tetrahydrochloride.

Analyses of the numbers of NeuN immunoreactive neurons and HIF-1 $\alpha$ immunoreactivity in CA1 and CA2/3 were conducted according to previously published methods $(9,19-21)$. In short, images of NeuN immunoreactive structures and HIF-1 $\alpha$ immunoreactive structures were captured using an AxioM1 light microscope at x20 magnification. To quantitatively analyze the numbers of NeuN immunoreactive neurons, the neurons were counted in $400 \mu \mathrm{m}^{2}$ at the middle of CA1 and CA2/3. To quantitatively analyze HIF-1 $\alpha$ immunoreactivity, the immunoreactive structures were captured at the same area of interest and calibrated into an array of $512 \times 512$ pixels. Finally, the density of the HIF-1 $\alpha$ immunoreactive structures was relatively evaluated using Adobe Photoshop version 8.0 and NIH Image J 1.59 software.

Statistical analysis. Data obtained in this study are expressed as the mean \pm SEM. We statistically analyzed the differences of the means between all groups by analysis of variance (ANOVA) with a post hoc Bonferroni's multiple comparison tests using SPSS program (version 18.0, IBM SPSS Statistics). For all statistical analyses, P-values $<0.05$ were considered statistically significant. 

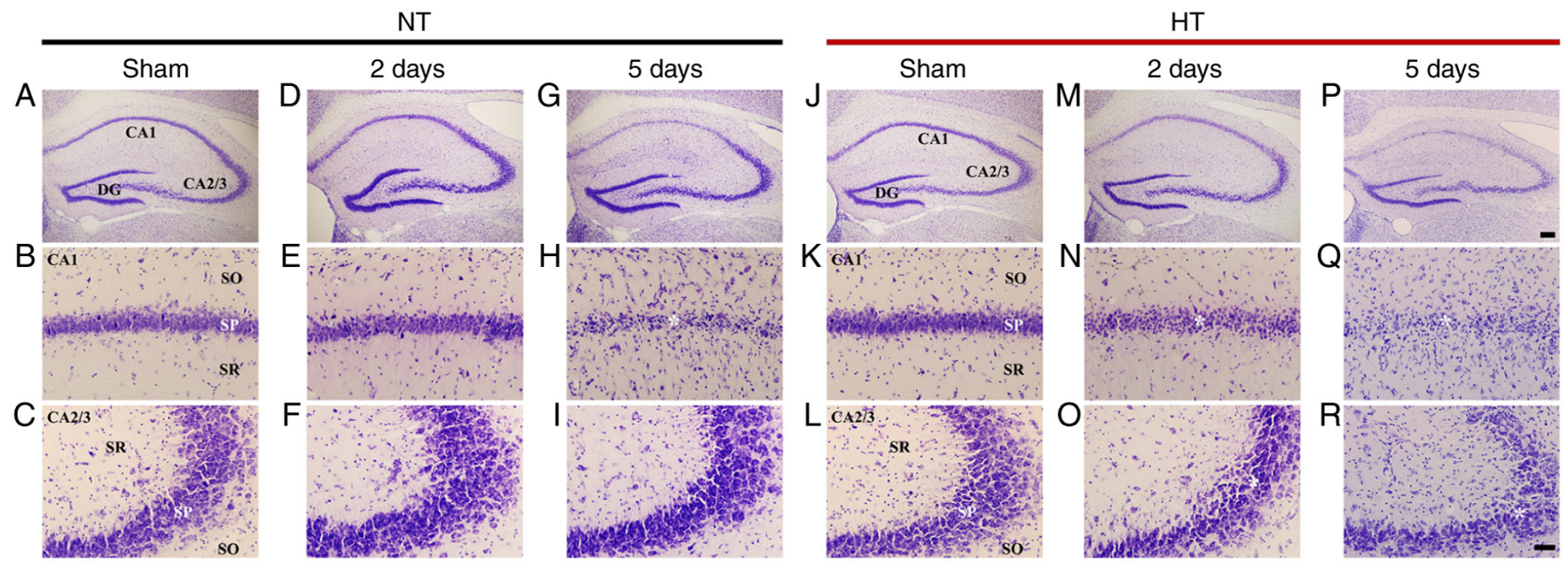

Figure 2. (A-R) Cresyl violet (CV) staining in CA1 and CA2/3 of the NT/sham (A-C), NT/ischemia (D-I), HT/sham (J-L), and HT/ischemia (M-R) groups on day 2 and 5 after TFI. CV-stained cells (asterisk in H) in the stratum pyramidale (SP) of CA1 of the NT/ischemia group were severely damaged on day 5 after TFI. In the HT/ischemia group, decreased stainability in CV-stained cells (asterisks in N, O) is shown in the SP of both CA1 and CA2/3 on day 2 after TFI. On day 5 after TFI, CV-stained cells (asterisks in Q, R) were apparently damaged in the SP of both CA1 and CA2/3. Scale bar, $200 \mu \mathrm{m}$ (upper row) and $50 \mu \mathrm{m}$ (middle and lower rows). DG, dentate gyrus; SO, stratum oriens; SR, stratum radiatum; NT, normothermia; HT, hyperthermia.

\section{Results}

\section{Differences in neuronal damage/death}

Findings by $C V$ histochemistry. CV-stained cells were easily identified in all subfields of the gerbil hippocampus of the NT/sham group. In particular, large pyramidal cells as principal neurons consisted of the stratum pyramidale (SP) (Fig. 2A-C). In the NT/ischemia group, the distribution of $\mathrm{CV}$-stained cells was not altered until 2 days after TFI in all subfields (Fig. 2D-F). However, on day 5 after TFI, CV-stained pyramidal cells of the SP were apparently damaged in CA1, but those located in CA2/3 were similar to those in the NT/sham group (Fig. 2G-I).

In the HT/sham group, the distribution of CV-stained cells in all subfields was not different from that in the NT/sham group (Fig. 2J-L). In the HT/ischemia group, on day 2 after TFI, most of the pyramidal cells in CA1 were weakly stained by $\mathrm{CV}$ (Fig. 2N), and CV-stained cells in CA2/3 were not significantly different from those of the HT/sham group (Fig. 2O). On day 5 after TFI, most of the CV-stained pyramidal cells in CA1 were severely damaged, and CA2/3 pyramidal cells showed weak CV staining or were damaged (Fig. 2P-R).

\section{Findings by FJB histofluorescence}

$C A 1$. FJB fluorescence staining revealed no FJB-stained degenerating (or dead) cells in the SP of CA1 in the NT/sham group (Fig. 3A-a). In the NT/ischemia group, FJB-stained cells were not found in the SP on days 1 and 2 after TFI (Fig. 3A-c and e). However, a significant increase in the number of FJB-stained cells $(36.2 \pm 2.7)$ was observed in the SP on day 5 after TFI (Fig. 3A-g and q).

In the HT/sham group, similar to the NT/sham group, FJB-stained cells were not found in the SP (Fig. 3A-i). In the HT/ischemia group, a few FJB-stained cells were detected in the SP on day 1 after TFI (Fig. 3A-k and q). In addition, abundant FJB-stained cells were found in the SP on days 2 and $5(30 \pm 1.5$ and $33.9 \pm 2.1$, respectively) after TFI (Fig. 3A-m, $o$ and $q)$.
$C A 2 / 3$. In the NT/sham group, FJB-stained cells were not observed in the SP of CA2/3 (Fig. 3B-a). In the NT/ischemia group, no FJB-stained cells were observed at any point in time after TFI in CA2/3 (Fig. 3B-c, e, g and q).

In the HT/sham group, FJB-stained cells were not detected in the SP of CA2/3 (Fig. 3B-i). In the HT/ischemia group, FJB-stained cells were not found in the SP on day 1 after TFI (Fig. 3B-k and q). However, many FJB-stained cells were found in the SP on day 2 after TFI $(29.4 \pm 2.8)$ (Fig. 3B-m and q). Furthermore, on day 5 after TFI, the number of FJB-stained cells was significantly increased

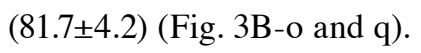

\section{Findings by NeuN immunohistochemistry}

CA1. In the NT/sham group, NeuN immunoreactivity was observed in the pyramidalcells of the SPinCA1 (Fig.3A-b).In the $\mathrm{NT} /$ ischemia groups, the distribution of NeuN-immunostained pyramidal cells in CA1 was not different from that of the NT/sham group on days 1 and 2 after TFI (Fig. 3A-d and f). However, on day 5 after TFI, NeuN-immunostained pyramidal cells were rarely observed in the SP. The percentage of remaining NeuN-immunostained pyramidal cells was $22.2 \%$ in the NT/sham group (Fig. 3A-h and r).

In the HT/sham group, the distribution of $\mathrm{NeuN}$ immunostained pyramidal cells was similar to that observed in the NT/sham group (Fig. 3A-j). In the HT/ischemia group, the NeuN immunoreactivity of NeuN-immunostained pyramidal cells was weak on day 1 after TFI (Fig. 3A-1). On days 2 and 6 after TFI, NeuN-immunostained pyramidal cells were significantly decreased (55.9 and $28.8 \%$ of sham, respectively) (Fig. 3A-n, p and r), showing that NeuN-immunostained pyramidal cells had significant morphological alterations (pyknotic and tangle-like appearance) on day 5 after TFI (Fig. 3A-p).

CA2/3. In the NT/sham group, NeuN-immunostained pyramidal cells were typically distributed in the SP of CA2/3 (Fig. 3B-b). In the NT/ischemia group, the distribution and 
A

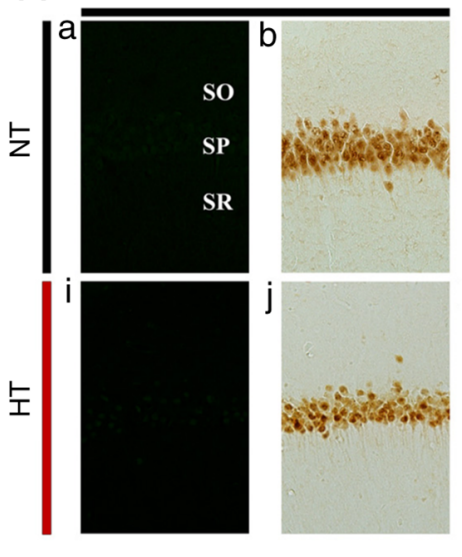

q

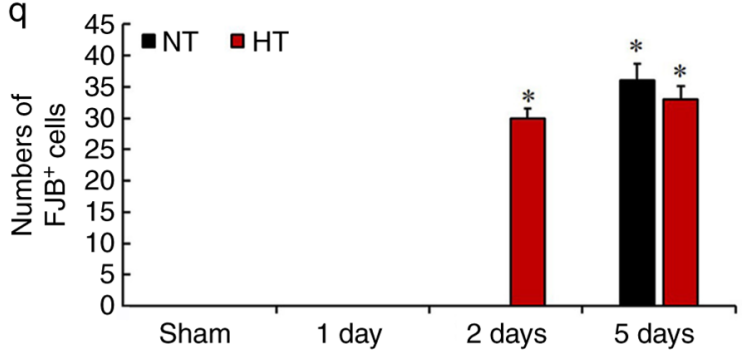

B
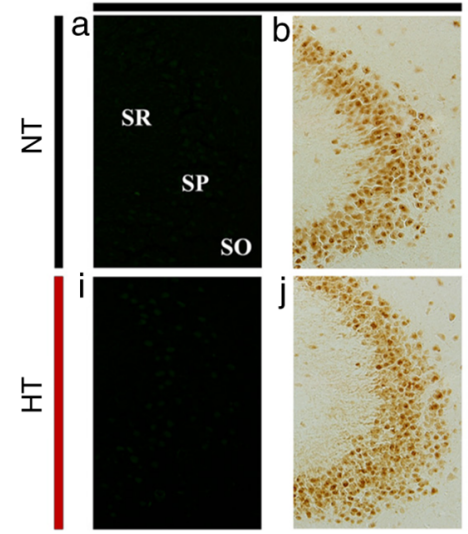

q

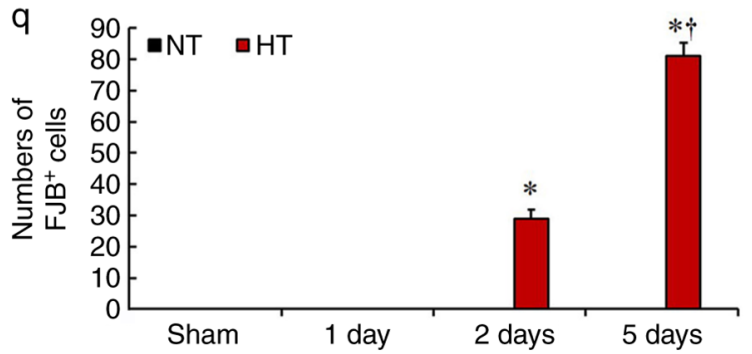

1 day
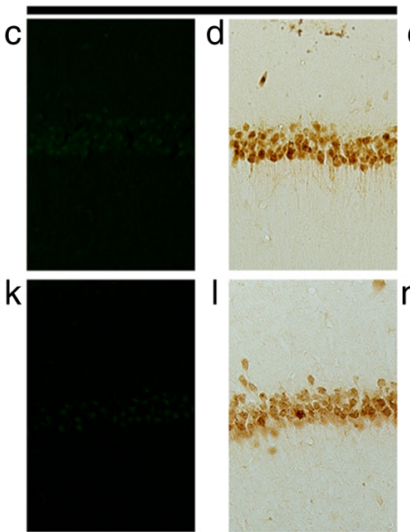

1 day
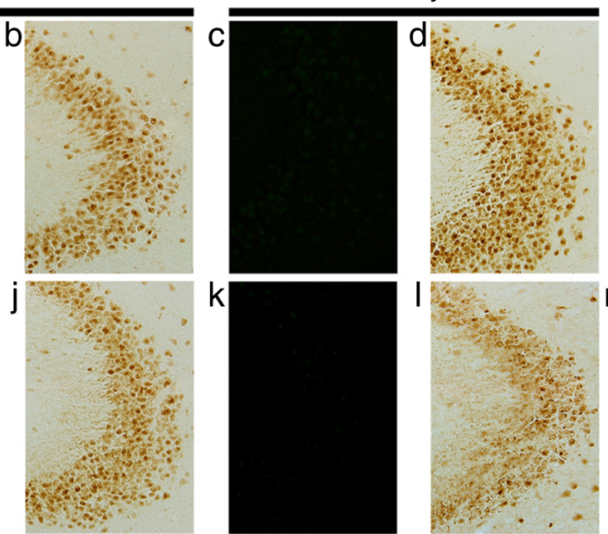
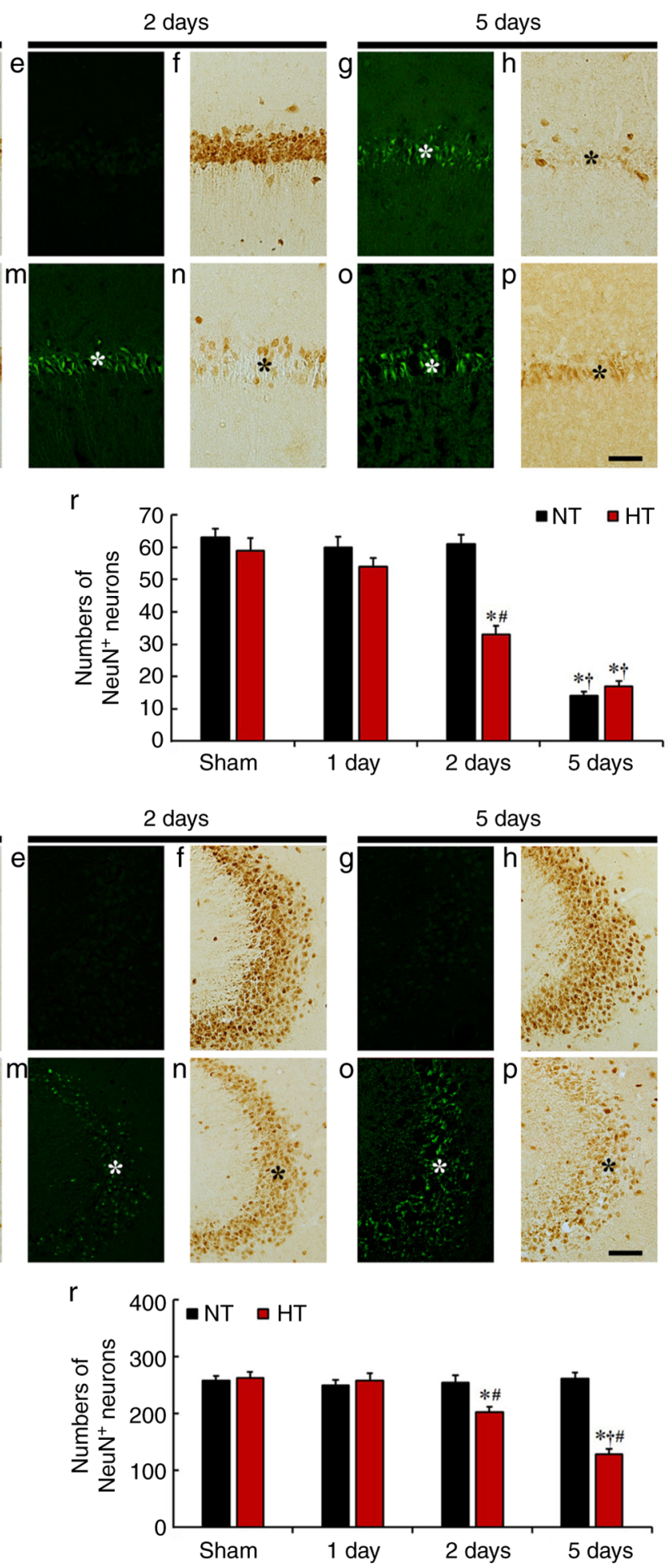

Figure 3. (A and B) Fluoro-Jade B (FJB) histofluorescence staining and anti-neuronal nuclei (NeuN) immunohistochemistry in CA1 (A) and CA2/3 (B) of the NT/sham (a and b), NT/ischemia (c-h), HT/sham (i and j) and HT/ischemia (k-p) groups on day 1, 2 and 5 after TFI. In the NT/ischemia group, many FJB-stained cells (white asterisk in A-g) were observed in the SP on day 5 after TFI in CA1, but not in CA2/3. However, in the HT/ischemia group, many FJB-stained cells (white asterisks in $\mathrm{m}$ and o) were detected in both CA1 and CA $2 / 3$ from 2 days after TFI. The numbers of NeuN-immunostained pyramidal cells of the NT/ischemia group were significantly reduced (black asterisk in A-h) only in CA1 on day 5 after TFI. In the HT/ischemia group, the numbers of NeuN-immunostained pyramidal cells were decreased (black asterisks in $\mathrm{n}$ and $\mathrm{p}$ ) in both CA1 and CA2/3 from 2 days after TFI. Scale bar, $50 \mu \mathrm{m}$. (q) Numbers of FJB-stained cells in CA1 (A) and CA2/3 (B). (r) Numbers of NeuN-immunostained cells in CA1 (A) and CA2/3 (B). "P<0.05 vs. NT/sham group; ${ }^{\dagger} \mathrm{P}<0.05$ vs. pre-time point group; ${ }^{\#} \mathrm{P}<0.05$ vs. NT/ischemia group. The bars indicate the means \pm SEM (n=7, respectively). Note: the use of only small letters indicates these panels in both $\mathrm{A}$ and $\mathrm{B}$.

number of NeuN-immunostained pyramidal cells were similar to those of the NT/sham group on days 1,2 and 5 after TFI (Fig. 3B-d, f, h and r).
In the HT/sham group, the distribution of NeuN-immunostained pyramidal cells was not different from that of the NT/sham group (Fig. 3B-j). In the HT/ischemia 

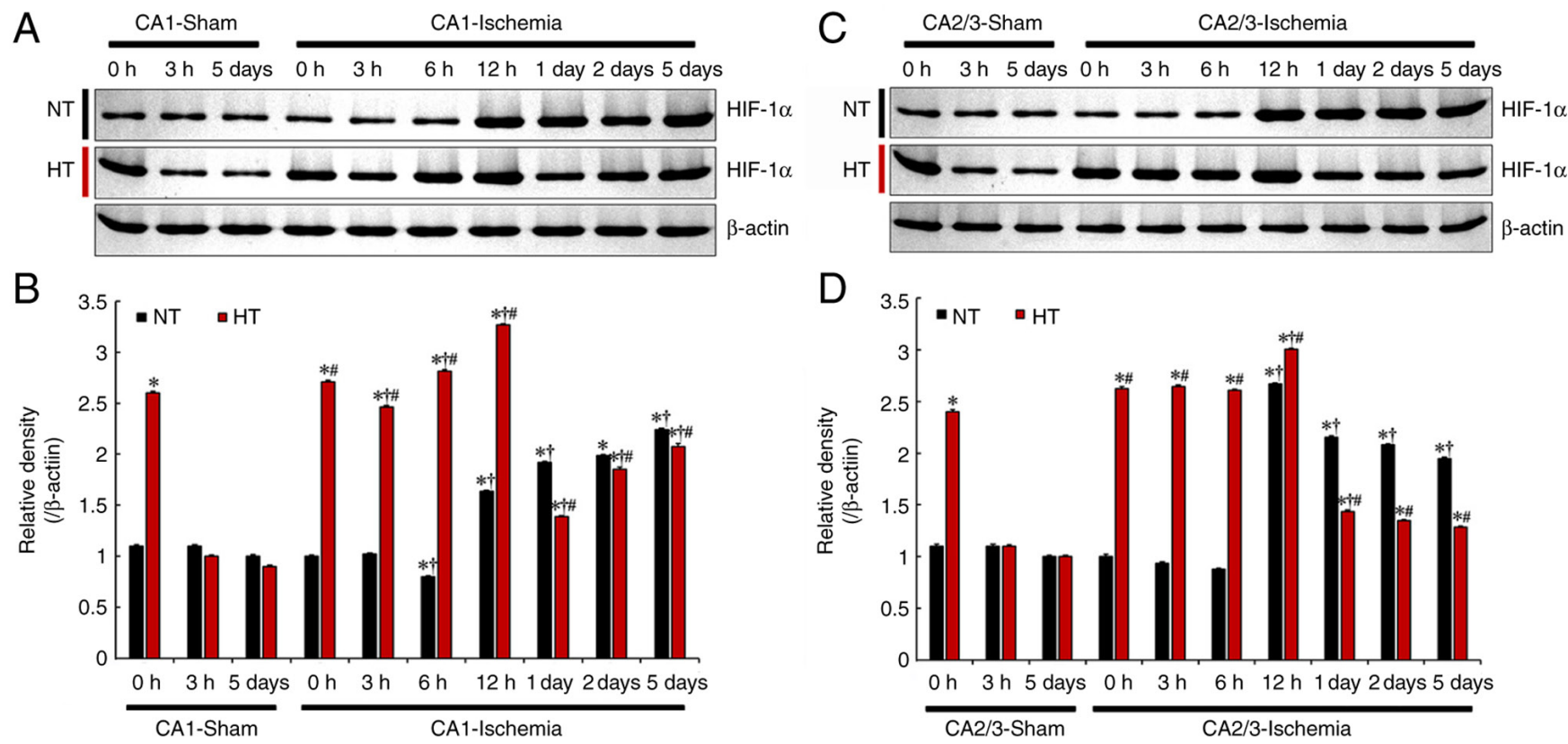

Figure 4. (A and C) Western blot analysis of HIF-1 $\alpha$ in CA1 (A) and CA2/3 (C) of the NT/sham, NT/ischemia, HT/sham, and HT/ischemia groups at 0,3 , 6 and $12 \mathrm{~h}, 1,2$ and 5 days after TFI. (B and D) Relative density of the immunoblot bands is represented. Protein levels of HIF-1 $\alpha$ normalized to $\beta$-actin in CA1 (B) and CA2/3 (D) " $\mathrm{P}<0.05$ vs. NT/sham group at $0 \mathrm{~h}$; ${ }^{ } \mathrm{P}<0.05$ vs. pre-time point group; ${ }^{~} \mathrm{P}<0.05$ vs. NT/ischemia group. The bars indicate the means $\pm \mathrm{SEM}$ $(\mathrm{n}=5$, respectively). HIF-1 $\alpha$, hypoxia-inducible factor $1 \alpha$; NT, normothermia; HT, hyperthermia; TFI, transient forebrain ischemia.

group, the NeuN immunoreactivity of the SP was slightly weak on day one after TFI (Fig. 3B-1). On days 2 and 5 after TFI, the number of NeuN-immunostained pyramidal cells was significantly reduced (78.7 and 49.9\%, respectively) compared to those in the HT/sham group (Fig. 3B-n, p and r).

\section{Differences in HIF-1 $\alpha$ protein level and immunoreactivity}

$H I F-1 \alpha$ protein level. The temporal pattern of HIF- $1 \alpha$ protein levels in CA1 and CA2/3 after TFI normothermia or hyperthermia were observed to be different, as shown in Fig. 4A and C. Small amounts of HIF-1 $\alpha$ protein were detected in CA1 and CA2/3 of the NT/sham group; the levels at 0 and $3 \mathrm{~h}$, and 5 days after TFI were not altered. In the NT/ischemia group, the level of HIF- $1 \alpha$ protein was significantly increased from $12 \mathrm{~h}$ after TFI (Fig. 4B and D). In the $\mathrm{HT} /$ sham group, at $0 \mathrm{~h}$ after TFI, HIF-1 $\alpha$ protein levels in CA1 and CA2/3 were significantly higher (236 and $218 \%$ of the NT/sham group, respectively) than that in the NT/sham group, and the increased HIF-1 $\alpha$ protein level was maintained until $6 \mathrm{~h}$ post-TFI, peaked at $12 \mathrm{~h}$ post-TFI, and after that decreased (Fig. 4B and D).

\section{HIF-1 1 immunoreactivity}

$C A 1$. In the NT/sham group, HIF-1 $\alpha$ immunoreactivity was hardly detected in CA1 pyramidal cells at 0 and $3 \mathrm{~h}$, and 5 days after TFI (Fig. 5A-a-c and C). In the NT/ischemia group, HIF-1 $\alpha$ immunoreactivity in the CA1 pyramidal cells was not significantly altered at 3 and $6 \mathrm{~h}$ after TFI (Fig. 5Ba-c and C), significantly increased at $12 \mathrm{~h}$ and 1 day after TFI (534.6 and $593.3 \%$ of the NT/sham group, respectively) (Fig. 5B-d, e and C), returned to baseline level (113.5\% of the NT/sham group) at 2 days after TFI (Fig. 5B-f and C), and again increased (208.7\% of the NT/sham group) at 5 days after TFI (Fig. 5B-g and C).
In the HT/sham group, HIF-1 $\alpha$ immunoreactivity was significantly increased (290.6\% of the NT/sham group) in the cytoplasm of the CA1 pyramidal cells at $0 \mathrm{~h}$ after sham TFI (Fig. 5A-d and C) and returned to the baseline from $3 \mathrm{~h}$ after TFI (Fig. 5A-e, f and C). In the HT/ischemia group, increased HIF- $1 \alpha$ immunoreactivity in the CA1 pyramidal cells was shown at $0,3,6$ and $12 \mathrm{~h}$ after TFI $(365.6,205.2$, 471.3 and $719.9 \%$ of the NT/sham group, respectively) (Fig. 5B-h-k and C). However, HIF-1 $\alpha$ immunoreactivity was dramatically decreased (15.4\% of the NT/ischemia group) at 1 day after TFI (Fig. 5B-1 and C), and, at 2 and 5 days after TFI, HIF-1 $\alpha$ immunoreactivity was similar to that observed in the NT/ischemia group (Fig. 5B-m, $n$ and C).

$C A 2 / 3$. In the NT/sham group, very weak HIF-1 $\alpha$ immunoreactivity was observed in CA2/3 pyramidal cells, showing that the immunoreactivity was not altered at 0 and $3 \mathrm{~h}$, and 5 days after TFI (Fig. 6Aa-c). In the NT/ischemia groups, HIF-1 $\alpha$ immunoreactivity in the CA2/3 pyramidal cells was slightly increased at 3 and 6 h (Fig. 6B-b, c and C), peaked (580.6\% of NT/sham group) at $12 \mathrm{~h}$ (Fig. 6B-d and C), and after that gradually decreased $(526.1 \%$ at 1 day, $411.8 \%$ at 2 days, and $412.6 \%$ at 5 days vs. the NT/sham group) until 5 days after TFI (Fig. 6Be-g and C).

In the HT/sham group, HIF-1 $\alpha$ immunoreactivity in the CA2/3 pyramidal cells was significantly increased $(250.3 \%$ of the NT/sham group) only at $0 \mathrm{~h}$ after sham TFI when compared with the NT/sham group (Fig. 6A-d and C). In the HT/ischemia group, HIF-1 $\alpha$ immunoreactivity in the CA2/3 pyramidal cells was gradually increased $(330.3 \%$ at $0 \mathrm{~h}$, $401.1 \%$ at $3 \mathrm{~h}$ and $385.6 \%$ at $6 \mathrm{~h}$ vs. the NT/sham group) (Fig. 6Bh-j and C), peaked (544.9\% of the NT/sham group) at $12 \mathrm{~h}$ (Fig. 6B-k and C), and after that significantly decreased (253.3\% at 1 day, $123.1 \%$ at 2 days, and $161.0 \%$ at 5 days vs. 

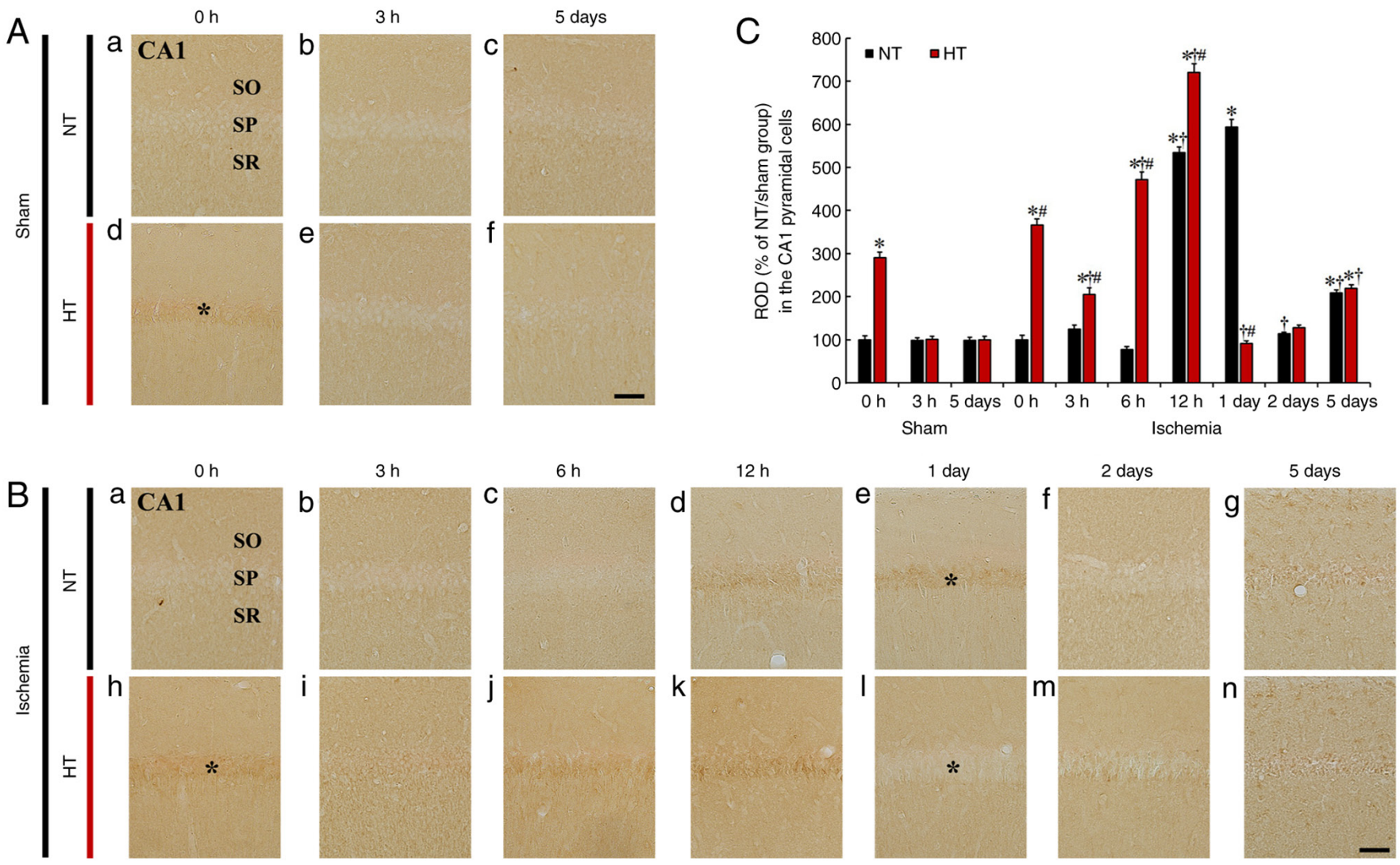

Figure 5. (A and B) HIF-1 $\alpha$ immunohistochemistry in CA1 of the (A) NT/sham (a-c), (B) NT/ischemia (a-g), (A) HT/sham (d-f) and (B) HT/ischemia (h-n) groups at $0,3,6$ and $12 \mathrm{~h}, 1,2$ and 5 days after TFI. In the NT/sham group, HIF-1 $\alpha$ immunoreactivity in the SP was very weak. In the NT/ischemia group, HIF-1 $\alpha$ immunoreactivity in the SP was significantly increased at $12 \mathrm{~h}$ and 1 day (asterisk), markedly reduced at 2 days and again increased at 5 days after TFI. In the HT/sham group, HIF-1 $\alpha$ immunoreactivity was very high (asterisk) at $0 \mathrm{~h}$ and similar to that of the NT/sham group from $3 \mathrm{~h}$ after sham TFI. In the HT/ischemia group, increased HIF-1 $\alpha$ immunoreactivity was gradually increased until $12 \mathrm{~h}$ after TFI and thereafter was lower (asterisk) than that in the HT/sham group. Scale bar, $50 \mu \mathrm{m}$. (C) Relative optical density (ROD) (\% of NT/sham group) of HIF-1 $\alpha$ immunoreactivity in CA1. ${ }^{~ P}<0.05$ vs. NT/sham group at $0 \mathrm{~h}$; ${ }^{\dagger} \mathrm{P}<0.05$ vs. pre-time point group; ${ }^{\prime} \mathrm{P}<0.05$ vs. NT/ischemia group. The bars indicate the means $\pm \mathrm{SEM}$ ( $\mathrm{n}=7$, respectively). HIF-1 $\alpha$, hypoxia-inducible factor $1 \alpha$; NT, normothermia; HT, hyperthermia; TFI, transient forebrain ischemia.

the NT/sham group) after TFI, showing that each immunoreactivity was significantly lower than that observed in the NT/ischemia group (Fig. 6Bl-n and C).

\section{Discussion}

Using a gerbil model of 5 min transient forebrain ischemia (TFI), we investigated the effect of TFI on neuronal death (loss) in the hippocampal CA1 and CA2/3 under hyperthermic conditions and the relationship between neuronal death and changes in hypoxia-inducible factor $1 \alpha$ (HIF-1 $\alpha$ ) expression following TFI under hyperthermia.

In the present study, TFI under normothermia induced selective death of pyramidal cells, which are principal neurons, in the CA1 on day 5 after TFI, but no loss of pyramidal cells occurred in CA2/3 on day 5 after TFI. This finding is consistent with the results of previous studies $(2,3,22)$. These results indicate that the pyramidal cells of CA1 are susceptible to brief transient ischemia, while the pyramidal cells of CA2/3 are resistant to brief transient ischemia, showing that there are differences in neuronal sensitivity to ischemic stress according to subfields in the hippocampus.

It has been reported that an elevation in body temperature (observed in more than $25 \%$ of patients with stroke) during or after ischemia induction is associated with more severe symptoms, poorer prognosis and mortality in patients with acute stroke $(23,24)$. It is known that an increase in core temperature during or after ischemic insults induces an increase in metabolic rate (25) and production of oxygen radicals (26), which may aggravate ischemic neuronal damage. In our present study, hyperthermia for $30 \mathrm{~min}$ before TFI (HT/ischemia group) resulted in the earlier death of pyramidal cells in CA1 on day 2 after TFI and induced death in CA2/3 pyramidal cells on day 2 after TFI, which was not observed in the NT/ischemia group. These results are similar to the findings of previous studies showing that, in rats and gerbils, ischemia-reperfusion under hyperthermic conditions induces more accelerated neuronal cell death in the hippocampal CA1 from approximately 1 day after transient ischemia (4-6), and disruption of ischemic resistance of pyramidal neurons in the hippocampal CA2/3 $(4,6)$ and granular cells in the hippocampal dentate gyrus (19). Furthermore, in a rat model of focal ischemic stroke, hyperthermia was found to increase infarct size and worsen behavioral outcomes when ischemic duration was maintained for more than $2 \mathrm{~h}$ (7). Based on these results, it can be suggested that hyperthermic conditions before and during transient ischemia augment and accelerate ischemic damage of pyramidal cells in the hippocampus compared to that under normothermia. 

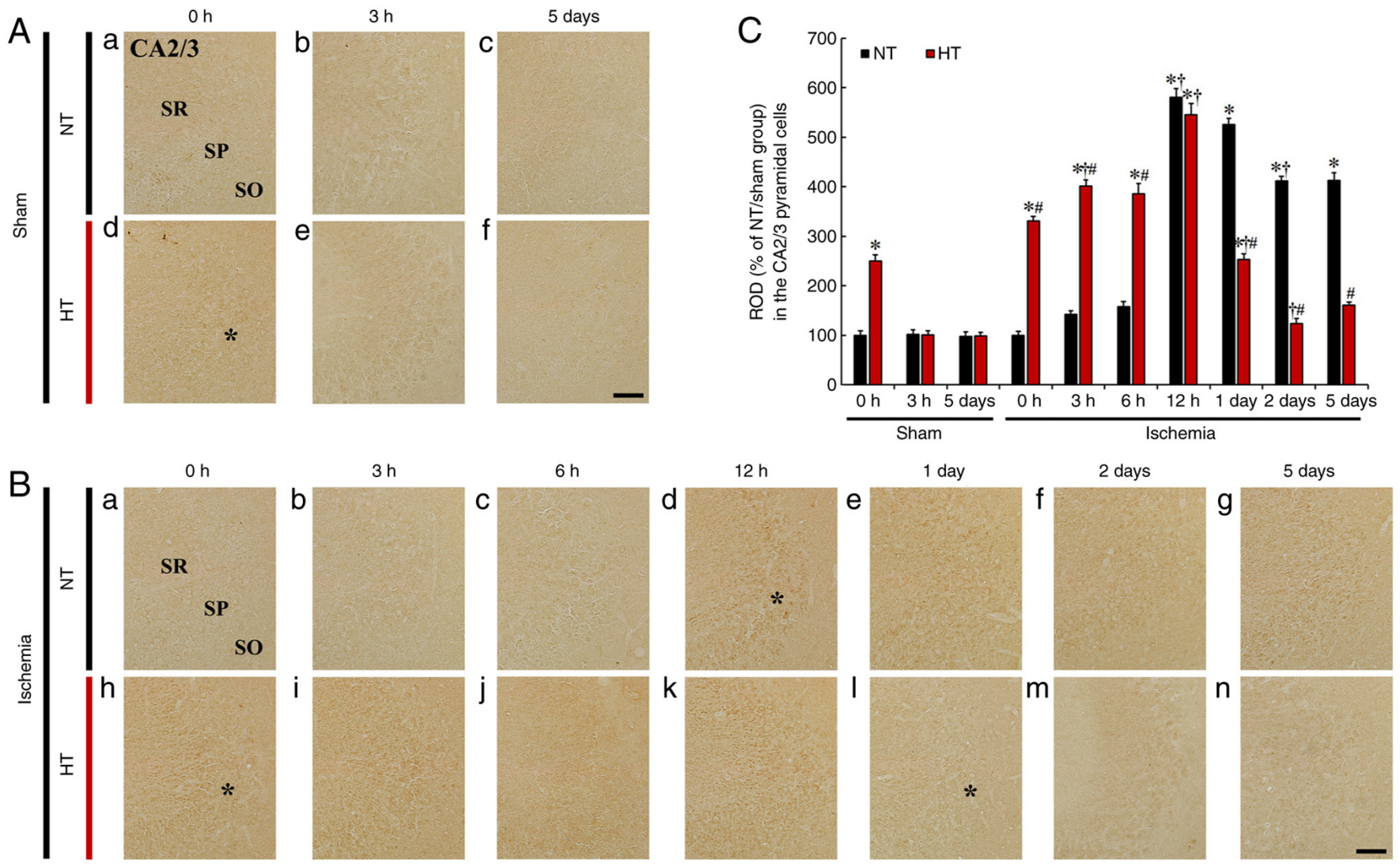

Figure 6. (A and B) HIF-1 $\alpha$ immunohistochemistry in CA2/3 of the (A) NT/sham (a-c), (B) NT/ischemia (a-g), (A) HT/sham (d-f) and (B) HT/ischemia (h-n) groups at $0,3,6$ and $12 \mathrm{~h}, 1,2$ and 5 days after TFI. In the NT/sham group, HIF-1 $\alpha$ immunoreactivity in the SP was very weak. In the NT/ischemia group, HIF-1 $\alpha$ immunoreactivity was significantly increased at $12 \mathrm{~h}$ (asterisk) and thereafter slightly reduced until 5 days after TFI. In the HT/sham group, HIF-1 $\alpha$ immunoreactivity was very high at $0 \mathrm{~h}$ and thereafter reduced to the level of the NT/sham group. In the HT/ischemia group, increased HIF-1 $\alpha$ immunoreactivity was continuously increased until 1 day, and thereafter HIF-1 $1 \alpha$ immunoreactivity was significantly decreased until 5 days after TFI. Scale bar, $50 \mu \mathrm{m}$. (C) Relative optical density (ROD) (\% of NT/sham group) of HIF-1 $\alpha$ immunoreactivity in CA2/3. ${ }^{*} \mathrm{P}<0.05 \mathrm{vs}$. NT/sham group at $0 \mathrm{~h}$; ${ }^{\circ} \mathrm{P}<0.05 \mathrm{vs}$. pre-time point group; ${ }^{*} \mathrm{P}<0.05$ vs. NT/ischemia group. The bars indicate the means \pm SEM ( $\mathrm{n}=7$, respectively). HIF-1 $\alpha$, hypoxia-inducible factor $1 \alpha$; NT, normothermia; HT, hyperthermia; TFI, transient forebrain ischemia.

It has been demonstrated that HIF-1 $\alpha$ is related to neuronal damage/death or survival in the brain following ischemic insults. Zhu et al reported that neuronal death in the hippocampal CA1 was observed, and HIF-1 $\alpha$ immunoreactivity was reduced following transient global ischemia in rats. They showed that the neuroprotective effect induced by hypoxia post-conditioning against ischemia was abolished by 2-methoxyestradiol (a specific HIF-1 $\alpha$ inhibitor) (11). In addition, Kopach et al reported the role of HIF-1 $\alpha$ in regulating neuronal protection. The activation of HIF- $1 \alpha$ decreased $\mathrm{Ca}^{2+}$-dependent excitotoxicity in CA1 neurons in situ through significant upregulation of endoplasmic reticulum $\mathrm{Ca}^{2+}$-ATPase mRNA levels after oxygen-glucose deprivation (27).

In the present study, we found that, in the NT/ischemia group, HIF-1 $\alpha$ expression was significantly elevated in the pyramidal cells of CA1 and CA2/3 at $12 \mathrm{~h}$, and 1 day after TFI the expression was differently decreased between CA1 and $\mathrm{CA} 2 / 3$. In CA1, the expression was significantly reduced on day 2 and increased again on day 5 after TFI, but, in CA2/3, the expression on days 2 and 5 after TFI was slightly decreased (significantly higher than that in CA1). Based on this finding, in the NT/ischemic group, survival of the CA2/3 pyramidal cells from TFI may be due to the difference in HIF-1 $\alpha$ expression on day 2 after TFI. This result is supported by a previous report showing that increased HIF-1 $\alpha$ immunoreactivity in ischemic CA1 was decreased before neuronal death in a gerbil model of TFI (9). In addition, Lushnikova et al reported that HIF-1 $\alpha$ mRNA levels were downregulated only in CA1 neurons, but not in CA3 neurons, along with the significant death of CA1 neurons in situ after oxygen-glucose deprivation (28), showing that the degree of HIF-1 $\alpha$ expression was different between the CA1 and CA2/3 pyramidal cells. Taken together, it can be speculated that a decrease in elevated HIF-1 $\alpha$ expression in the hippocampal CA1 is closely related to ischemic neuronal cell death and persistence of high HIF-1 $\alpha$ expression in CA2/3 may contribute to ischemic resistance of $\mathrm{CA} 2 / 3$ pyramidal neurons.

On the other hand, under hyperthermic conditions, the basal level of HIF-1 $\alpha$ expression at $0 \mathrm{~h}$ in the HT/sham and HT/ischemia groups was significantly increased in both CA1 and CA2/3 pyramidal cells when compared to the NT/sham and NT/ischemia groups. Similar to this early increase in HIF-1 $\alpha$ expression under hyperthermia in the present study, an earlier increase in HIF-1 $\alpha$ expression was reported in previous studies using an animal model that caused relatively severe damage compared to $5 \mathrm{~min}$ of TFI under normothermia. Upregulated HIF-1 $\alpha$ was observed immediately after $10 \mathrm{~min}$ of TFI in the rat hippocampal CA1 region (11) and at $30 \mathrm{~min}$ after hypoxia-ischemia injury in the mouse cortex (29). In the 
present study, HIF-1 $\alpha$ expression peaked at $12 \mathrm{~h}$ in both CA1 and CA2/3 pyramidal cells of the HT/ischemia group and then significantly decreased on day 1 after TFI (just before neuronal cell death), showing earlier neuronal death despite the initial high expression of HIF-1 $\alpha$ as compared with the NT/ischemia group. Although the exact reason for the early increase in HIF-1 $\alpha$ expression remains unclear, it may be due to a protective mechanism against greater damage, or another mechanism may be involved in hyperthermia-induced severe neuronal damage. Therefore, based on our and previous studies, it is likely that the reduction of increased HIF-1 $\alpha$ expression in the hippocampus precedes ischemic neuronal cell death under normothermic and hyperthermic conditions.

In summary, the present study showed that pyramidal cells in CA1 and CA2/3 had differences in ischemia sensitivity under normothermia, and the relatively high resistance of CA2/3 pyramidal cells may be closely related to the maintenance of high expression of HIF- $1 \alpha$ after TFI. In addition, hyperthermia accelerated ischemic neuronal death in both CA1 and CA2/3, showing that hyperthermia-induced neuronal death is closely related to a reduction in increased HIF-1 $\alpha$ expression in both ischemic CA1 and CA2/3. Therefore, we should consider the environment or conditions of the brain in which ischemic insults occur because ischemic damage in the brain is not the same in all patients with ischemic stroke.

\section{Acknowledgements}

The authors would like to acknowledge Mr. Seung Uk Lee and Ms. Hyun Sook Kim for their technical assistance in this study.

\section{Funding}

This research was supported by Basic Science Research Program through the National Research Foundation of Korea (NRF) funded by the Ministry of Education (NRF-2021R1A2C1094224, NRF-2020R1F1A1052380, and NRF-2020R1I1A1A01070897).

\section{Availability of data and materials}

The data presented in this study are available on request from the corresponding author.

\section{Authors' contributions}

Conceptualization of the study concept was achieved by MHW and JHA. Investigations in regards to western blot analysis and immunohistochemistry were carried out by TKL, DWK and HS. The study methodology, use of the software, validation of the data collected and supervision of the study were the responsibility of JCL, HIK, MCS, JHC, JHP, CHL, JHP and JHC. Data curation was carried out by TKL, DWK and JHA. Writing of the original draft was carried out by TKL, DWK, MHW and JHA. Writing of the review and editing were MHW and JHA. Project administration was the responsibility of TKL DWK, MHW and JHA. Funding acquisition was carried out by JHA, MHW and TKL. All authors have read and agreed to the published version of the manuscript.

\section{Ethics approval and consent to participate}

Experimental procedures for the present study were approved by the Institutional Animal Care and Use Committee at Kangwon National University (approval no. KW-200113-1). The procedures of handling and caring animals conformed to the guidelines of the current international laws and policies (NIH Guide for the Care and Use of Laboratory Animals, The National Academies Press, 8th ediiton, 2011).

\section{Patient consent for publication}

Not applicable.

\section{Competing interests}

The authors declare that they have no competing interests.

\section{Authors' information}

Professor Moo-Ho Won: ORCID: 0000-0002-7178-6501; Professor Ji Hyeon Ahn, ORCID: 0000-0002-5304-0714.

\section{References}

1. Wang $X$ and Michaelis EK: Selective neuronal vulnerability to oxidative stress in the brain. Front Aging Neurosci 2: 12, 2010.

2. Kirino T: Delayed neuronal death in the gerbil hippocampus following ischemia. Brain Res 239: 57-69, 1982.

3. Yu DK, Yoo KY, Shin BN, Kim IH, Park JH, Lee CH, Choi JH, Cho YJ, Kang IJ, Kim YM and Won MH: Neuronal damage in hippocampal subregions induced by various durations of transient cerebral ischemia in gerbils using Fluoro-Jade B histofluorescence. Brain Res 1437: 50-57, 2012.

4. Minamisawa H, Smith ML and Siesjo BK: The effect of mild hyperthermia and hypothermia on brain damage following 5 , 10 and 15 min of forebrain ischemia. Ann Neurol 28: 26-33, 1990.

5. Dietrich WD, Busto R, Valdes I and Loor Y: Effects of normothermic versus mild hyperthermic forebrain ischemia in rats. Stroke 21: 1318-1325, 1990.

6. Kim MJ, Cho JH, Park JH, Park JH, Ahn JH, Tae HJ, Cho GS, Yan BC, Hwang IK, Lee CH, et al: Impact of hyperthermia before and during ischemia-reperfusion on neuronal damage and gliosis in the gerbil hippocampus induced by transient cerebral ischemia. J Neurol Sci 348: 101-110, 2015.

7. de Jonge JC, Wallet $\mathrm{J}$ and van der Worp HB: Fever worsens outcomes in animal models of ischaemic stroke: A systematic review and meta-analysis. Eur Stroke J 4: 29-38, 2019.

8. Albadari N, Deng S and Li W: The transcriptional factors HIF-1 and HIF-2 and their novel inhibitors in cancer therapy. Expert Opin Drug Discov 14: 667-682, 2019.

9. Lee JC, Tae HJ, Kim IH, Cho JH, Lee TK, Park JH, Ahn JH, Choi SY, Bai HC, Shin BN, et al: Roles of HIF-1 $\alpha$, VEGF, and $\mathrm{NF}-\kappa \mathrm{B}$ in ischemic preconditioning-mediated neuroprotection of hippocampal CA1 pyramidal neurons against a subsequent transient cerebral ischemia. Mol Neurobiol 54: 6984-6998, 2017.

10. Long Q, Fan C, Kai W, Luo Q, Xin W, Wang P, Wang A, Wang Z, Han R, Fei Z, et al: Hypoxia inducible factor-1 $\alpha$ expression is associated with hippocampal apoptosis during epileptogenesis. Brain Res 1590: 20-30, 2014.

11. Zhu T, Zhan L, Liang D, Hu J, Lu Z, Zhu X, Sun W, Liu L and $\mathrm{Xu}$ E: Hypoxia-inducible factor $1 \alpha$ mediates neuroprotection of hypoxic postconditioning against global cerebral ischemia. J Neuropathol Exp Neurol 73: 975-986, 2014.

12. Shi $\mathrm{H}$ : Hypoxia inducible factor 1 as a therapeutic target in ischemic stroke. Curr Med Chem 16: 4593-4600, 2009.

13. Chatzi C, Schnell E and Westbrook GL: Localized hypoxia within the subgranular zone determines the early survival of newborn hippocampal granule cells. Elife 4: e08722, 2015.

14. Xing J and Lu J: HIF-1 $\alpha$ activation attenuates IL- 6 and TNF- $\alpha$ pathways in hippocampus of rats following transient global ischemia. Cell Physiol Biochem 39: 511-520, 2016. 
15. National Research Council (US) Committee for the Update of the Guide for the Care and Use of Laboratory Animals: Guide for the Care and Use of Laboratory Animals. 8th edition. National Academies Press, Washington, DC, 2011.

16. Kim B, Ahn JH, Kim DW, Lee TK, Kim YS, Shin MC, Cho JH, Kim YM, Park JH, Kang IJ, et al: Transient forebrain ischemia under hyperthermic condition accelerates memory impairment and neuronal death in the gerbil hippocampus by increasing NMDAR1 expression. Mol Med Rep 23: 2562021

17. Ohk TG, Ahn JH, Park YE, Lee TK, Kim B, Lee JC, Cho JH, Park JH, Won $\mathrm{MH}$ and Lee $\mathrm{CH}$ : Comparison of neuronal death and expression of TNF- $\alpha$ and MCT4 in the gerbil hippocampal CA1 region induced by ischemia/reperfusion under hyperthermia to those under normothermia. Mol Med Rep 22: 1044-1052, 2020.

18. Kanda I: Exotic animal formulary 4 edition. Can Vet J 56: 736, 2015

19. Kim DW, Cho JH, Cho GS, Kim IH, Park JH, Ahn JH, Chen BH, Shin BN, Tae HJ, Hong S, et al: Hyperthermic preconditioning severely accelerates neuronal damage in the gerbil ischemic hippocampal dentate gyrus via decreasing SODs expressions. J Neurol Sci 358: 266-275, 2015

20. Yang GE, Tae HJ, Lee TK, Park YE, Cho JH, Kim DW, Park JH, Ahn JH, Ryoo S, Kim YM, et al: Risperidone treatment after transient ischemia induces hypothermia and provides neuroprotection in the gerbil hippocampus by decreasing oxidative stress. Int J Mol Sci 20: 4621, 2019.

21. Lee JC, Cho JH, Lee TK, Kim IH, Won MH, Cho GS, Shin BN, Hwang IK, Park JH, Ahn JH, et al: Effect of hyperthermia on calbindin-D 28k immunoreactivity in the hippocampal formation following transient global cerebral ischemia in gerbils Neural Regen Res 12: 1458, 2017.

22. Bartsch T and Wulff P: The hippocampus in aging and disease: From plasticity to vulnerability. Neuroscience 309: 1-16, 2015.
23. Azzimondi G, Bassein L, Nonino F, Fiorani L, Vignatelli L, Re G and D'Alessandro R: Fever in acute stroke worsens prognosis: A prospective study. Stroke 26: 2040-2043, 1995.

24. Reith J, Jørgensen HS, Pedersen PM, Nakayama H, Raaschou HO, Jeppesen LL and Olsen TS: Body temperature in acute stroke: Relation to stroke severity, infarct size, mortality, and outcome. Lancet 347: 422-425, 1996.

25. Busija DW, Leffler CW and Pourcyrous M: Hyperthermia increases cerebral metabolic rate and blood flow in neonatal pigs. Am J Physiol 255 (2 Pt 2): H343-H346, 1988.

26. Corbett D and Thornhill J: Temperature modulation (hypothermic and hyperthermic conditions) and its influence on histological and behavioral outcomes following cerebral ischemia. Brain Pathol 10: 145-152, 2000.

27. Kopach O, Maistrenko A, Lushnikova I, Belan P, Skibo G and Voitenko N: HIF-1 $\alpha$-mediated upregulation of SERCA2b: The endogenous mechanism for alleviating the ischemia-induced intracellular $\mathrm{Ca}(2+)$ store dysfunction in $\mathrm{CA} 1$ and $\mathrm{CA} 3$ hippocampal neurons. Cell Calcium 59: 251-261, 2016.

28. Lushnikova I, Orlovsky M,Dosenko V, Maistrenko A and Skibo G: Brief anoxia preconditioning and HIF prolyl-hydroxylase inhibition enhances neuronal resistance in organotypic hippocampal slices on model of ischemic damage. Brain Res 1386: 175-183, 2011.

29. Jeon GW, Sheldon RA and Ferriero DM: Hypoxia-inducible factor: Role in cell survival in superoxide dismutase overexpressing mice after neonatal hypoxia-ischemia. Korean J Pediatr 62: 444-449, 2019. International (CC BY-NC-ND 4.0) License. 